\title{
J-curve revisited: cardiovascular benefits of moderate alcohol use cannot be dismissed
}

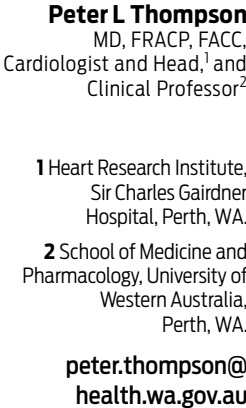

doi: 10.5694/mjal2.10922

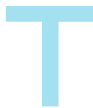

here is universal agreement that the alcoholrelated problems of violence, motor vehicle fatalities and injuries, family disruption and significant ill health are major challenges for the Australian community, ${ }^{1,2}$ as they are worldwide. ${ }^{3}$ Concerted action is required to address this. ${ }^{4}$ In response, Australia's National Health and Medical Research Council (NHMRC) produced a thoroughly researched document in $2009,^{5}$ which updated its 2001 Australian alcohol guidelines. ${ }^{6}$

The NHMRC guidelines, important as a basis for alcohol policy in Australia, were unbalanced on an important issue: the potential health benefits of one to two standard drinks per day were reported incompletely. In a 118-page report, the potential health benefits of low doses of alcohol were dealt with in less than half a page. It made no comment on the extent of the literature showing that people consuming one to two standard drinks per day have a lower risk of cardiovascular outcomes than abstainers. At the time of the 2009 NHMRC guidelines, there had been over 60 high-quality large-scale studies on alcohol use on over a million subjects and associations with over 94500 deaths., ${ }^{7,8}$ This extensive database has widely influenced alcohol policy in many countries and has been the basis of statements on alcohol intake and cardiovascular disease from most national and international health advisory bodies (Box 1).

The NHMRC guidelines appeared to dismiss this extensive evidence base with the statement that "some recent studies have found no significant cardioprotective or allcause associations" and suggested that any effect may have been overestimated. It relied for this assessment on a single publication that queried the prior research on the apparent cardioprotective effect of low to moderate alcohol intake. ${ }^{9}$ It has already influenced other Australian bodies in their alcohol policy; it is repeated without any update from recent literature in the 2011 position statement of the Cancer Council of Australia, which highlights the potential for low doses of alcohol to cause cancer. ${ }^{10}$

The dismissal of the cardiovascular and overall mortality benefits of low to moderate intake of alcohol has the potential to bias the debate about the dangers of alcohol in our community. The recent campaigns to publicise the potential for alcohol to cause cancer ${ }^{11,12}$ have been launched without providing balancing information that drinking alcohol within the limits set by the NHMRC guidelines may have cardiovascular and overall mortality benefits.

\section{The J-shaped curve}

Multiple studies from the late $1970 \mathrm{~s}^{13}$ onwards have reached a general consensus: people consuming one to

\section{Summary \\ - While the Australian National Health and Medical Research Council guideline document of 2009 on reducing health risks from drinking alcohol provided sensible advice for public policy on alcohol, it appeared to dismiss the cardiovascular benefits of low to moderate consumption. \\ - Undue prominence was given to a hypothesis from a single research group that the well documented J-curve relationship of lower risk of ischaemic heart disease events with low to moderate intake alcohol consumption may have been due to a misclassification of drinking patterns. \\ - The misclassification hypothesis suggested that the higher risks among abstainers may have been due to the inclusion of high-risk subjects who had become abstainers later in life. \\ - Recent studies have separated recent abstainers from lifetime abstainers and the misclassification hypothesis has not been confirmed as an explanation for the J-shaped curve. \\ - The J-shaped relationship between alcohol consumption and cardiovascular risk has been studied and confirmed in multiple studies; while it complicates the formulation of public policy on alcohol consumption, it cannot be dismissed.}

two standard drinks per day appear to have a lower cardiovascular event rate than persons abstaining from alcohol; a relationship described as a J-shaped (or Ushaped) curve. ${ }^{14}$ Estimates of the risk reduction associated with one to two standard drinks per day compared with those abstaining from alcohol have ranged from $20 \%$ to $30 \%{ }^{7}$ Over many years, there have been criticisms of the interpretation of this association, the two most prominent being the possibilities of misclassification of the reference group and confounding from uncontrolled other lifestyle factors. This brief review makes no comment on the important issue of whether the association can be construed as a causal relationship. This question is unlikely to be resolved without a randomised trial. ${ }^{15}$ Many feasible biological pathways for a possible mechanism of benefit of alcohol have been proposed, ${ }^{16}$ and beneficial trends in biomarkers with low to moderate intake of alcohol strengthen the case $\mathrm{e}^{17}$ but do not prove causation per se. The main focus of this review is whether misclassification of drinking habits explains the J-shaped relationship between alcohol consumption and cardiovascular disease.

\section{Misclassification of drinking habits}

In stating that "some recent studies have found no significant cardioprotective or all-cause associations", the 2009 
1 Statements from international medical bodies on the relationship between low to moderate alcohol intake and cardiovascular disease

Organisation
American Heart Association

Royal Australasian College of Physicians

American Diabetes Association

World Health Organization

\section{Relevant quote}

"There are more than 60 prospective studies that suggest an inverse relation between moderate alcoholic beverage consumption and coronary heart disease"

"The injury and chronic disease burden associated with risky or high risk levels of alcohol consumption are offset by a decrease in the burden of cardiovascular disease produced by minimal alcohol consumption"

"In individuals with diabetes, light to moderate alcohol intake (1 or 2 drinks per day; 15 to $30 \mathrm{~g}$ alcohol) is associated with a decreased risk of CV disease, which does not appear to be due to an increase in HDL cholesterol"

"Moderate drinking, that is 1 or 2 units a day, may offer some protection from coronary heart disease"

"Light to moderate drinking can have a beneficial impact on morbidity and mortality for ischaemic heart disease and ischaemic stroke"

European Society of Cardiology "recent meta-analyses carried out in large series of subjects and published in prestigious journals show that 1-2 drinks per day may be in fact, beneficial"
Year

2001

http://my.americanheart.org/professional/

StatementsGuidelines/ByTopic/TopicsA-C/StatementGuideline-Topics-A C_UCM_322827_Article.jsp (accessed Mar 2013)

2005

Royal Australasian College of Physicians. Alcohol policy: using evidence for better outcomes. Sydney: RACP, 2005

2006 Bantle JP, Wylie-Rosett J, Apovian CM, et al. Nutrition recommendations and interventions for diabetes - 2006: a position statement of the American Diabetes Association. Diabetes Care 2006; 29: 2140-2157

Current www.bhf.org.uk/alcohol (accessed Mar 2013)

Current http://www.who.int/substance_abuse/publications/ global_alcohol_report/msbgsruprofiles.pdf (accessed Mar 2013)

Current http://www.escardio.org/communities/councils/ccp/ejournal/volume9/Pages/low-doses-of-alcohol-ClementDenis.aspx (accessed Mar 2013)
NHMRC report relied on a 2006 meta-analysis by Fillmore and colleagues ${ }^{9}$ and an expansion of their views in a 2007 review. ${ }^{18}$ Their meta-analysis presented a reinterpretation of previous studies, developing and expanding the "sick quitter" hypothesis first developed in 1988 by Shaper and colleagues. ${ }^{19}$ This interpretation broadly proposes that misclassification of drinking habits may have resulted in more coronary events in the abstainer reference group because it included ex-drinkers with an increased cardiovascular risk because of ill health or increasing age. The sick quitter hypothesis was criticised in several studies carried out during the 1990s. Fillmore and colleagues reexamined the hypothesis using their own methodology to exclude all studies with misclassification errors in the abstainer reference group. However, they took such a strict approach that they identified only seven studies on total mortality and two on coronary heart disease mortality free of the misclassification errors. In this small number of studies, the evidence base remaining failed to confirm the J-shaped relationship between alcohol consumption and cardiovascular events. The conclusion from their analysis was that "future prospective studies should use far greater precision in their assessment of drinking behavior and abstinence" ${ }^{18}$ Recent studies confirm the suspicion that that there is indeed a difference between former drinkers and lifetime abstainers in their pattern of ischaemic heart disease (IHD) incidence and mortality, but no evidence that this differentiation makes a substantive difference to the relationship between lower levels of cardiovascular events with a low to moderate intake of alcohol. ${ }^{20}$ While it is possible that younger adults with a disability may choose an abstinent lifestyle and suffer a higher mortality in adulthood from their disability and not their abstinence, this accounts for only a very small proportion of nondrinkers and is unlikely to contribute substantially to the Jshaped relationship. ${ }^{21}$

Since the misclassification version of the sick quitter hypothesis was put forward in 2006 and embraced in the 2009 NHMRC guidelines, the relationship and the possibility of misclassification in the abstainer group has been re-examined in several recent major meta-analyses. Corrections for possible misclassification error have been attempted in more recent studies, and none support misclassification as an interpretation of the J-shaped curve.

\section{Patients with incident cardiovascular disease}

Three recent meta-analyses on incident cardiovascular disease do not support the misclassification hypothesis. A BMJ meta-analysis published in late 2011 represents the most complete meta-analysis to date. ${ }^{22}$ It found no differences in the extent of relative risk reduction in cardiovascular disease mortality when classification adjustments were made to address the sick quitter misclassification hypothesis. Of the 4235 studies considered and 84 studies involving over one million people included in the final analysis, the pooled estimates showed a lower risk of allcause mortality for drinkers compared with non-drinkers (relative risk, 0.87; 95\% CI, 0.83-0.92). The meta-analysis did not include a graphic representation of the J-curve, but it did report the dose relationships that can be graphed as shown in Box 2, confirming the J-shaped relationship between alcohol intake and cardiovascular morbidity and mortality. A variety of cardiovascular outcomes were examined. The relative risks were no different when active low to moderate drinkers were compared with all abstainers versus lifetime abstainers. The relative risk of experiencing a cardiovascular outcome among those with a light to moderate intake when the reference group was limited to lifetime abstainers was 0.82 ( $95 \% \mathrm{CI}, 0.78-0.86)$. The relative risks for incident coronary heart disease were even lower.

This meta-analysis has been criticised by Fillmore and colleagues for including many of the studies that they had previously ranked as statistically inadequate, ${ }^{23}$ but two other more recent meta-analyses have specifically addressed the question of misclassification. An analysis comparing lifetime abstainers with former drinkers showed that inclusion of former drinkers in the reference group may influence estimates of IHD morbidity, but not estimates of IHD mortality. ${ }^{20}$ Another meta-analysis, 
2 Meta-analysis showing the J-shaped relationship between cardiovascular mortality and alcohol intake based on 84 studies involving over a million people

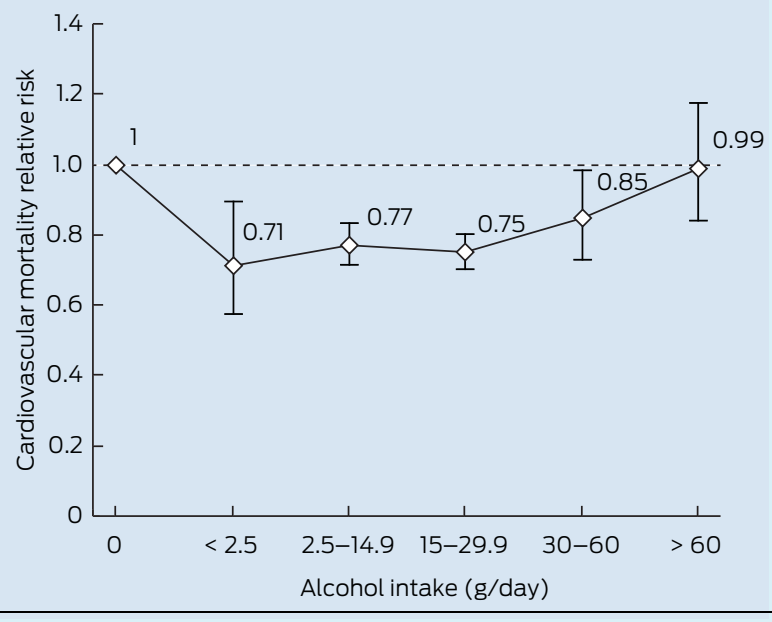

Redrawn from data in Ronksley et al..22

which completely excluded any studies that may have included former drinkers or sick quitters and restricted itself to lifetime abstainers, assessed 38627 IHD events (mortality or morbidity) among 957684 participants. ${ }^{24}$ This thorough analysis showed differential risk curves for sex and end point, but the overall conclusion was that there was a cardioprotective effect with low to moderate alcohol intake. The trends for men and women at the low to

3 Lifetime abstainers compared with occasional and low to moderate consumers of alcohol, showing associations with lower rates of ischaemic heart disease (IHD) morbidity and mortality in men and reduced morbidity in women
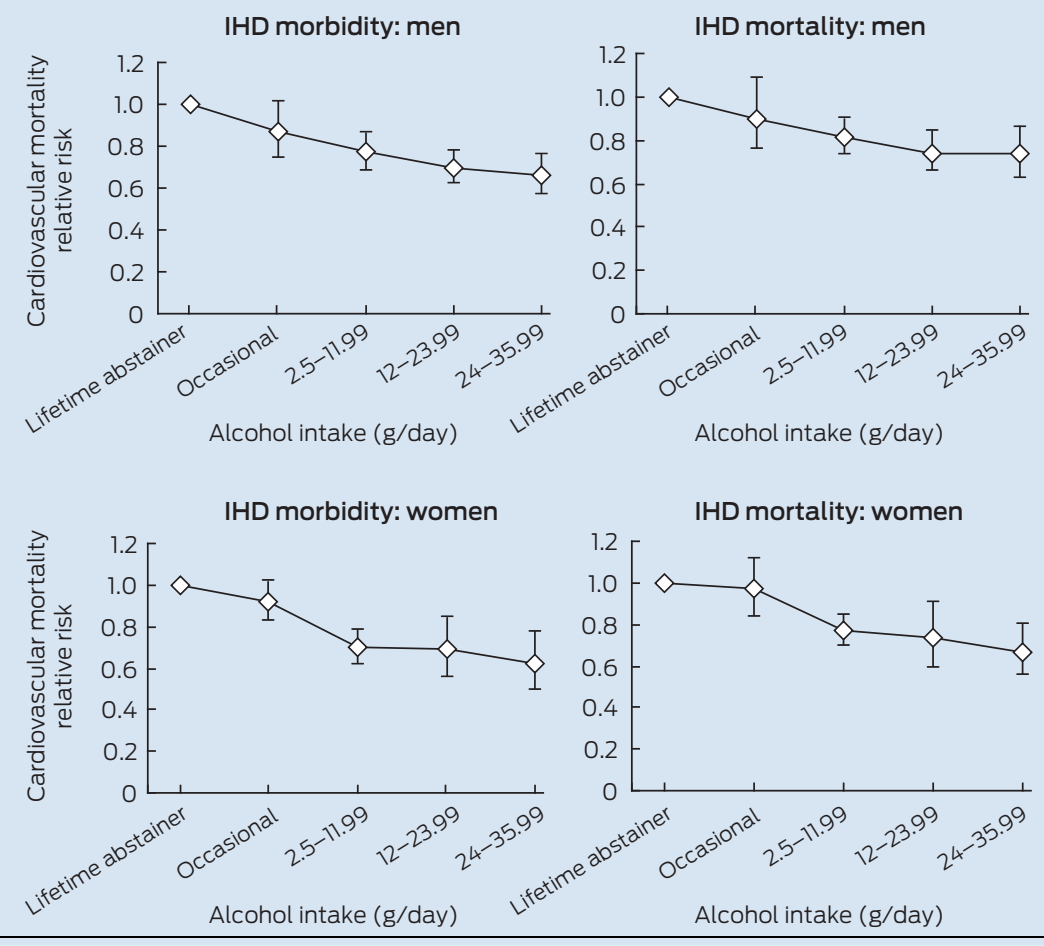

Redrawn from data in Roerecke and Rehm. ${ }^{24}$ moderate end of the scale of daily consumption presented in this analysis are shown in Box 3. When all levels of consumption were analysed with lifetime abstainers as the reference group, the typical J-shaped pattern emerged (Box 2).

\section{Patients with established cardiovascular disease}

Studies of people who already have cardiovascular disease do not support the misclassification hypothesis. In 2010, a large meta-analysis examined the effect of alcohol consumption in patients with established cardiovascular disease, finding no difference in its conclusions when studies with lifetime abstainers were analysed separately from all abstainers. ${ }^{25}$ Seven studies comprising 12819 patients with cardiovascular disease provided detail on doseresponse relationships between alcohol consumption and cardiovascular outcomes. This analysis confirmed the Jshaped relationship for alcohol intake for patients with established cardiovascular disease.

\section{Regular low to moderate alcohol intake compared with binge drinking}

The J-shaped association between alcohol consumption and cardiovascular disease applies to regular low to moderate alcohol intake but not to binge drinking. Two recent meta-analyses have demonstrated a lower risk of cardiovascular disease with a regular daily intake of a low to moderate dose of alcohol and a higher risk with infrequent binge drinking. ${ }^{26,27}$

\section{Confounding and causation}

This brief review is an update of the evidence on whether a regular low to moderate intake of alcohol is associated statistically with a lower risk of coronary heart disease. It does not comment on whether the lower risk implies that a causal relationship is simply because of lifestyle factors unrelated to alcohol intake. While coronary heart disease is the only disease that shows the J-shaped relationship with alcohol, ${ }^{28}$ the question of whether a low to moderate intake of alcohol is truly protective is unlikely to be resolved without a randomised controlled trial. ${ }^{15}$ While many explanations for a possible mechanism of benefit of alcohol in coronary heart disease have been proposed, ${ }^{16}$ they do not prove a causal relationship per se. Extensive metaanalysis of 21 biomarkers strengthened the case for a causal relationship, concluding that favourable changes in cardiovascular biomarkers "provide indirect pathophysiological support for a protective effect of moderate alcohol use on coronary heart disease" ${ }^{17}$ While the observational evidence is inconclusive that a low to moderate intake of alcohol is cardioprotective, much of the data on the adverse health effects of alcohol depend on a similar quality of evidence, including the conclusion that it is a carcinogen. ${ }^{29}$

\section{Conclusion}

The evidence for a J-shaped relationship between alcohol consumption and cardiovascular outcomes is extensive. 
The possibility that the shape of the curve may be due simply to a misclassification of drinking habits, as suggested in the 2009 NHMRC guidelines, has not been supported by recent studies. The overall impact on community health of a $20 \%-30 \%$ lower rate of coronary disease in light to moderate drinkers is likely to be substantial. ${ }^{30}$ While the J-shaped curve complicates the formulation of public policy on alcohol, ${ }^{31}$ it cannot be dismissed.

Competing interests: I am owner and director of Thompson Estate vineyard and winery, Margaret River, WA.

Provenance: Not commissioned; externally peer reviewed.

1 Chikritzhs T, Catalano P, Stockwell T, et al. Australian alcohol indicators, 1990 2001. Patterns of alcohol use and related harms for Australian States and Territories. Perth: National Drug Research Institute, Curtin University of Technology, 2003. http://ndri.curtin.edu.au/local/docs/pdf/naip/ naipaaifullreport.pdf (accessed Feb 2013)

2 Australian Institute of Health and Welfare. 2007 National Drug Strategy Household Survey: first results. Canberra: AlHW, 2008. (AlHW Cat. No. PHE 98; Drug Statistics Series No. 20.) http://www.aihw.gov.au/publication-detail/ ?id=6442468084 (accessed Feb 2013).

3 WHO Expert Committee on Problems Related to Alcohol Consumption. Second report. World Health Organ Tech Rep Ser 2007; (944): 1-53, 55-57.

4 Casswell S, Thamarangsi T. Reducing harm from alcohol: call to action. Lancet 2009; 373: 2247-2257.

5 National Health and Medical Research Council. Australian guidelines to reduce health risks from drinking alcohol. Canberra: NHMRC, 2009. http:// www.nhmrc.gov.au/guidelines/publications/ds10 (accessed Feb 2013).

6 National Health and Medical Research Council. Australian alcohol guidelines. Health risks and benefits [revoked]. Canberra: Commonwealth of Australia, 2001. http://www.nhmrc.gov.au/_files_nhmrc/publications/attachments/ ds9.pdf (accessed Feb 2013).

7 Di Castelnuovo A, Costanzo S, Barnardo V, et al. Alcohol dosing and total mortality in men and women: an updated meta-analysis of 34 prospective studies. Arch Intern Med 2006; 166: 2437-2445.

8 Rimm EB, Klatsky A, Grobbee D, Stampfer MJ. Review of moderate alcohol consumption and reduced risk of coronary heart disease: is the effect due to beer, wine, or spirits? BMJ 1996; 312: 731-736.

9 Fillmore K, Kerr W, Stockwell T, et al. Moderate alcohol use and reduced mortality risk: systematic error in prospective studies. Addict Res Theory 2006; 14, 101-132.

10 Winstanley MH, Pratt IS, Chapman K, et al. Alcohol and cancer: a position statement from Cancer Council Australia. Med J Aust 2011; 194: 479-482.

11 Cresswell A. Alcohol blamed for cancers. The Australian 2011; 2 May. http:// www.theaustralian.com.au/news/nation/alcohol-blamed-for-cancers/storye6frg6nf-1226048008362 (accessed Mar 2012).
12 Miller B. Alcohol linked to 20pc of breast cancer cases. ABC News [internet] 2011; 2 May. http://www.abc.net.au/news/2011-05-02/alcohol-linked-to20pc-of-breast-cancer-cases/2697618 (accessed Mar 2012).

13 St Leger AS, Cochrane AL, Moore F. Factors associated with cardiac mortality in developed countries with particular reference to the consumption of wine. Lancet 1979; 1: 1017-1020.

14 Connor J. The life and times of the J-shaped curve. Alcohol Alcohol 2006; 41: 583-584.

15 Jackson R, Broad J, Connor J, Wells S. Alcohol and ischaemic heart disease: probably no free lunch. Lancet 2005; 366: 1911-1912.

16 Kloner RA, Rezkalla SH. To drink or not to drink? That is the question. Circulation 2007: 116: 1306-1317.

17 Brien SE, Ronksley PE, Turner BJ, et al. Effect of alcohol consumption on biological markers associated with risk of coronary heart disease: systematic review and meta-analysis of interventional studies. BMJ 2011; 342: d636.

18 Fillmore KM, Stockwell T, Chikritzhs T, et al. Moderate alcohol use and reduced mortality risk: systematic error in prospective studies and new hypotheses. Ann Epidemiol 2007; 17 (5 Suppl): S16-S23.

19 Shaper A, Wannamethee G, Walker M. Alcohol and mortality in British men: explaining the U-shaped curve. Lancet 1988; 2: 1267-1273.

20 Roerecke $M$, Rehm J. Ischemic heart disease mortality and morbidity rates in former drinkers: a meta-analysis. Am J Epidemiol 2011; 173: 245-258.

$21 \mathrm{Ng}$ Fat L, Shelton N. Associations between self-reported illness and nondrinking in young adults. Addiction 2012; 107: 1612-1620.

22 Ronksley PE, Brien SE, Turner BJ, et al. Association of alcohol consumption with selected cardiovascular disease outcomes: a systematic review and meta-analysis. BMJ 2011; 342: d671.

23 Stockwell T, Greer A, Fillmore K, et al. How good is the science [letter]? BMJ 2012; 344: e2276.

24 Roerecke M, Rehm J. The cardioprotective association of average alcohol consumption and ischaemic heart disease: a systematic review and metaanalysis. Addiction 2012; 107: 1246-1260.

25 Costanzo S, Di Castelnuovo A, Donati MB, et al. Alcohol consumption and mortality in patients with cardiovascular disease: a meta-analysis. J Am Coll Cardiol 2010; 55: 1339-1347.

26 Bagnardi V, Zatonski W, Scotti L, et al. Does drinking pattern modify the effect of alcohol on the risk of coronary heart disease? Evidence from a metaanalysis. J Epidemiol Community Health 2008; 62: 615-619.

27 Roerecke M, Rehm J. Irregular heavy drinking occasions and risk of ischemic heart disease: a systematic review and meta-analysis. Am J Epidemiol 2010; 171: 633-644.

28 Corrao G, Bagnardi V, Zambon A, La Vecchia C. A meta-analysis of alcohol consumption and the risk of 15 diseases. Prev Med 2004; 38: 613-619.

29 IARC Working Group on the Evaluation of Carcinogenic Risks to Humans. Alcohol consumption and ethyl carbamate. IARC Monogr Eval Carcinog Risks Hum 2010; 96: 3-1383.

30 Wells S, Broad J, Jackson R. Alcohol consumption and its contribution to the burden of coronary heart disease in middle-aged and older New Zealanders: a population-based case-control study. N Z Med J 2004; 117: U793.

31 Hall W. What place, if any, does information on putative cardioprotective effects of moderate alcohol use have in safer drinking guidelines? Drug Alcohol Rev 2012; 31: 194-197. 